\title{
Politisches System und \\ New Public Management in \\ Primarschulen im \\ internationalen Vergleich
}

\section{Franziska Vogt}

Ziel dieser Untersuchung der Schulreformen und ihrer Umsetzung ist die Erkundung des Zusammenspiels zwischen der länderübergreifenden Ideologie des New Public Management und den lokalen politischen Systemen und professionellen Kulturen. England und die Schweiz erlauben einen besonders interessanten Vergleich, weil sich ihre politischen Systeme stark unterscheiden - zentralisiert und im Majorzsystem in England, dezentralisiert und auf parteienübergreifenden Konsens bauend in der Schweiz (Pollitt \& Bouckaert, 2000). Dieser Artikel geht der Frage nach, wie sich die Zusammenarbeit unter den Lehrpersonen und die Rolle der Schulleitung verändern. Die Ergebnisse aus vier Fallstudien weisen darauf hin, dass das politische System die Ausprägung der Schulreform nicht nur auf der Ebene des Bildungssystem beeinflusst, sondern auch auf der Ebene der einzelnen Schule, besonders in den schulischen Entscheidungsprozessen und im Machtgefüge zwischen Schulleitung, lokalen Schulbehörden und Lehrpersonen.

\section{Einleitung}

New Public Management beeinflusst Reformen im öffentlichen Sektor in vielen Regionen der Welt. Internationale Vergleichsstudien ermöglichen es, das $\mathrm{Zu}$ sammenspiel dieser international diskutierten Ideologie, der Reform in Bildungssystemen und der lokalen Umsetzung in Primarschulen zu untersuchen. In dieser Studie werden die zwei Bildungssysteme von England innerhalb Grossbritanniens und des Kantons Luzern innerhalb der Schweiz verglichen. Diese beiden Bildungssysteme unterscheiden sich stark in ihrer Grösse: Im Vergleich zum Kanton Luzern besuchen 150-mal mehr Schülerinnen und Schüler englische Primarschulen. Entscheide über Schulreformen werden auf dieser Ebene gefällt. In ihrer Analyse mehrerer europäischer Länder zeigen Pollitt \& Bouckaert (2000), dass das schweizerische und das britische politische System zwei Extreme repräsentieren in Bezug auf zwei wesentliche Faktoren: auf das System zur Wahl der Exekutive und auf das Ausmass an Dezentralisation. Während Pollitt und 
Bouckaert (2000) bei den andern analysierten europäischen Ländern verschiedene Kombinationen dieser zwei Faktoren fanden, bilden Grossbritannien und die Schweiz die zwei entgegengesetzten Pole: Majorzsystem für die Exekutiven und zentralisierte Entscheidung in Grossbritannien, Exekutiven unter Beteiligung aller stärkeren Parteien und Föderalismus in der Schweiz.

New Public Management geht davon aus, dass der öffentliche Sektor von der Privatwirtschaft lernen soll, unter anderem im Bereich des Finanzmanagements und der Personalführung, und dass der öffentliche Sektor nach dem Modell der freien Marktwirtschaft restrukturiert werden soll, beispielsweise durch die Schaffung von Wettbewerb und die Betonung der Kundenorientierung (Alford, 1993; Clarke \& Newman, 1997; Deem, 1998; Farnham \& Horton, 1993; Flynn, 1999). New Public Management beruht wesentlich auf bestimmten Normen und Werten und kann nicht nur als ein Set von Management-Techniken verstanden werden, die wie ein Zaubermittel eingesetzt werden (Trowler, 1998). New Public Management ist damit beides gleichzeitig, «a delivery system and a vehicle for change» (Ball, 1998, S. 123). New Public Management wurde zuerst in den angelsächsischen Ländern eingeführt (Pollitt \& Bouckaert, 2000); innerhalb Europas hat Grossbritannien früh und umfassend entsprechende Reformen umgesetzt (Ferlie, Ashburner, Fitzgerald \& Pettigrew, 1996). Die Aspekte des New Public Management werden in den einzelnen Ländern unterschiedlich betont: In England wird auf die Einführung des Marktes im öffentlichen Sektor, neue Rechenschaftssysteme und die Standardisierung der Bildung Wert gelegt; in der Schweiz steht die Zusammenarbeit, Schulentwicklung und die Ablösung des früheren kollegialen Systems der Schulkoordination durch Schulleitungen im Zentrum.

Dieser Beitrag beruht auf Forschungsergebnissen des Projektes «Kontextualisierung von New Public Management in Primarschulen in England und der Schweiz: Die Wahrnehmungen der Lehrpersonen und ihre Erfahrungen mit Schulreform, Organisationskultur und Zusammenarbeit» (Vogt, 2002). Die Forschung beinhaltet Fallstudien von vier staatlichen Primarschulen in beiden Ländern. Für die Schweiz wurden Schulen im Kanton Luzern ausgewählt, da im Kanton Luzern durch neue gesetzliche Grundlagen die Schulreformen für alle Schulen Gültigkeit haben, während in anderen Kantonen häufig eine Projektstruktur mit Versuchsschulen gewählt wurde. Die Pilotstudie zu diesem Forschungsprojekt zeigte, dass die besondere Situation von Versuchsschulen die Schulkultur stark prägt (Vogt, 2001). In England betreffen die Schulreformen zugleich alle öffentlichen Schulen, so dass sich Schulen aus dem Kanton Luzern besser für den Vergleich eignen. In der Auswahl der Schulen wurde auf gewisse strukturelle Ähnlichkeiten geachtet: alle Schulen sind öffentliche Schulen, haben ihre eigene Schulleitung und Schulpflege, die ausschliesslich für diese Primarschule zuständig sind. Die Grösse der Schule wurde als Variable, welche die Zusammenarbeit der Lehrpersonen prägt, in den Vergleich miteinbezogen. Jeweils eine Schule mit etwa 100 Schülerinnen und Schüler sowie eine Schule mit 250- 
300 Schülerinnen und Schülern wurden ausgewählt. Im Folgenden werden die Schulen als $E g$ für «Englisch gross», $E k$ für «Englisch klein», $S g$ für «Schweiz gross» und Sk für «Schweiz klein» bezeichnet. Alle Namen sind Pseudonyme.

Im Forschungsprojekt wurden ethnografische Methoden verwendet. Nach Skeggs (1994) zeichnet sich dieser Ansatz insbesondere durch längere Feldforschung aus, wobei der Teilnahme der Forschenden im alltäglichen Kontext und der Entwicklung der Beziehungen zwischen den Beteiligten und ihrer Reflexion grosse Bedeutung zu kommt; ethnografische Forschung in diesem Verständnis hat häufig die Kultur einer Organisation oder einer Gruppe zum Fokus und analysiert diese als Mikrokosmos umfassenderer struktureller Prozesse (Skeggs, 1994). Während ethnografische Forschung traditionell auf einen Kontext konzentriert ist, wurde für diese Forschungsfrage das Design der multi case study gewählt. Dadurch ist die Feldforschung in der einzelnen Schule zeitlich begrenzter, die Möglichkeiten des Vergleichs jedoch besser (Schofield, 2000); das Multi Case Study Design hat den Vorteil, dass länder- und schulspezifische Kulturen eher unterschieden werden können. Der ständige Wechsel zwischen Feldforschung und Analyse, welcher den ethnografischen Ansatz generell auszeichnet, ist für den internationalen Vergleich hilfreich (Vogt, 2002). Empirische Verallgemeinerungen auf alle Schulen sind aufgrund ethnografischer Fallstudien nicht möglich, theoretische Übertragbarkeit ist damit jedoch nicht ausgeschlossen (Gomm, Hammersley \& Foster, 2000). Die Fallstudien erlauben, theoretische Überlegungen zu entwickeln, die für andere Schulen von Bedeutung sein können, da möglicherweise ähnliche Prozesse ablaufen.

In diesem Projekt wurden die folgenden Methoden eingesetzt: Beobachtung, informelle Gespräche und halbstrukturierte Interviews sowie die Analyse von Dokumenten der lokalen Schulen und die Analyse von Reformdokumenten der Kantone und der nationalen Behörden. Die Validität der Daten und Analyse wurde durch eine sorgfältige, auf theoretischen Kriterien beruhende Auswahl der Fallstudienschulen, Triangulation durch die verschiedenen Methoden und den mehrfachen Wechsel zwischen Feldforschung, Analyse und Verifizierung der Analyse in der nächsten Feldforschung sichergestellt.

\section{Politisches System und Schulreform}

Das politische System der beiden Länder, insbesondere die Kontraste zwischen Zentralisation und Dezentralisation und von Konsens mehrerer Parteien oder Mehrheitsparteienregierung prägen die Einführung, Schwerpunktsetzung und Umsetzung der Schulreformen auf der Ebene des Bildungssystems.

Das föderalistische schweizerische politische System mit einer Regierungsform, die alle stärkeren politischen Kräfte umfasst, macht eine umfassende und schnelle Umsetzung von New Public Management-Reformen schwierig (Pollitt $\&$ Bouckaert, 2000). Im Kanton Luzern wurde die New Public Management- 
Reform als Projekt initiiert. Die Erkenntnisse und Erfahrungen dieses Projektes flossen in das neue Volksschulgesetz ein:

Kantonsvertreter: Im Volksschulbereich mussten wir zuerst entwickeln, was wir eigentlich wollen. Weil, wenn wir jetzt da etwas übernehmen aus dem angelsächsischen Raum, oder auch von fortschrittlichen deutschen Bundesländern, dann stehen wir möglicherweise einfach vor der Tatsache, dass wir wissen, was wir wollen, aber alle andern dem nicht folgen können. [...] Das war der Punkt. Da hat man dann gesagt, da muss man ja zuerst den Boden vorbereiten. [...] Auf diesem Hintergrund haben wir dann das Projekt "Schulen mit Profil» lanciert, wo wir [...] einen besonderen Weg gewählt haben: Die Trägerschaft sind nicht wir alleine, sondern [...] alle [...] massgeblich an der Volksschule Beteiligten, nämlich die Lehrpersonen, der Verband der Schulpflegepräsidentinnen und-präsidenten und wir [...] wenn wir hier Ideen entwickeln und die Ideen immer in dieser Dreierträgerschaft [...] zur Übereinstimmung gebracht werden müssen, dann hat das auch eine Chance, nachber im Gesetz (Interview Transkript).

Die politische Kultur in Grossbritannien hingegen ist durch das Kräftemessen zwischen den zwei grossen Parteien charakterisiert. Die Regierung einer Partei ist für eine Zeitdauer von etwa fünf Jahren gewählt und versucht in dieser Zeit ihre politischen Ziele zu realisieren. Schulreform wird vom britischen Erziehungsministerium gesteuert (Kogan, 1986). Die Reform des öffentlichen Sektors nach New Public Management-konzepten und damit auch die Schulreform wurden in einer Reihe von Gesetzesänderungen zunächst unter der Regierung der Conservatives in den Achtziger- und frühen Neunzigerjahren eingeführt. New Labour hat weitere von New Public Management geprägte Reformen eingebracht (Bottery, 2000). Die Interpretation von New Public Management und die Begründung der Reformen unterscheidet sich bei den beiden politischen Parteien zwar in Details, die kontinuierliche, weit reichende Restrukturierung des Bildungssystems nach New Public Management-Grundsätzen ist jedoch deutlich sichtbar. Entscheidende Reformen waren:

- Einführung eines Quasi-Marktsystems mit Pro-Kopf-Beiträgen, elterlicher Schulwahl und Veröffentlichung der Testabschlüsse je Schule,

- Dezentralisierung von Aufgaben auf die Ebene der Schule

- gleichzeitig Zentralisierung durch neue Kontrollsysteme wie standardisierte Tests, Inspektionen durch eine halbprivate Organisation, Leistungsbeurteilung und Leistungslohn sowie die Einführung eines nationalen Lehrplanes.

Im Zuge der Reform wurden in England die Schulräte der einzelnen Schulen gestärkt. In ihre Verantwortung gehört Personal, Umsetzung des Lehrplans, Qualitätsmanagement, Gebäude und Finanzen. Untersuchungen zeigen eine Veränderung in der Kultur der Zusammenarbeit der Schulbehörden insofern, als Schulräte verstärkt die Schulleitungen und Lehrpersonen kontrollieren (Deem, Brehony \& Heath, 1995). Im Gegensatz zu den Schweizer Schulpflegen sind englische Schulräte nur für eine Schule zuständig und weniger mit den politischen und administrativen Strukturen der lokalen Gemeinde vernetzt. Gemein- 
destrukturen sind in England tendenziell wenig ausgeprägt. Hingegen ist das Konzept des Kommunitarismus einflussreich. Der Kommunitarismus bildet eine der ideologischen Wurzeln des New Public Management und ist besonders bei Politikerinnen und Politikern der Neuen Linken beliebt (Bottery, 2000; Exworthy \& Halford, 1999). Als Beispiel zeigt die kürzlich eingeführte obligatorische Namensänderung diese Dynamik. "County Schools», also staatliche Schulen des Bezirks, mussten ihren Namen ändern zu "Community Schools». Diese Veränderung in der Namensgebung zeigt eine Veränderung weg vom Staat und seiner öffentlichen Verwaltung, die auf den politischen und demokratischen Prozessen der verschiedenen Ebenen wie Gemeinde, Bezirk und Nation aufbaut. "Community» ist eine undefinierbare Gruppe in Bezug auf bürokratische oder politische Strukturen. "Community» wird eher als eine Gruppe von Personen verstanden, die Werte und moralische Vorstellungen teilen und in bestimmten Aufgaben gemeinsame Verantwortung übernehmen (Bottery, 2000). In der Realität ist jedoch die Betonung auf New Public Management und Community ein Paradox. Die auf New Public Management gründenden Reformen wie beispielsweise Wettbewerb zwischen Schulen und freie elterliche Schulwahl schwächen die Verbindung zwischen der lokalen Gemeinschaft und ihrer Schule. Kinder einer Schule reisen von anderen lokalen "Communities» her an, während Kinder, die in der Nähe einer Schule wohnen, in eine andere Schule gehen.

\section{Veränderungen im schulischen Machtgefüge}

Die konkrete Umsetzung der Reformen in den einzelnen Schulen ist eng mit der Organisationskultur der Schulen verbunden. Zum einen erhebt New Public Management den Anspruch, die Organisationskultur im öffentlichen Sektor zu verändern, zum Beispiel mit einer verstärkten Orientierung an den als Kundinnen und Kunden verstandenen Nutzenden des öffentlichen Dienstes (Clarke \& Newman, 1993). Zum andern beeinflusst, wie im Folgenden gezeigt werden wird, die Organisationskultur die Umsetzung der Schulreformen. Organisationskultur wird als gemeinsames Verständnis von Organisationsprozessen und als gemeinsames Bedeutungssystem definiert (Schein, 1991). Die Organisationskultur einer Schule wird laufend durch die Beteiligten aktiv realisiert, interpretiert und erneuert. Das Machtgefüge ist für die Ausprägung der Organisationskultur entscheidend (Alvesson \& Deetz, 1999; Mill \& Mills, 2000). Die Reformen des New Public Management haben zu bedeutenden Veränderungen der Rolle der Schulleitung in beiden Ländern geführt. Schulleitungen werden als die wichtigsten Träger der Reformen auf der Ebene der Schule gesehen (Lowe, 1998).

Englische Schulleitungen als machtvolle Autoritäten In den Aussagen der englischen Lehrpersonen zeigt sich, wie sich der Führungs- 
stil der Schulleitungen verändert hat. In der Vergangenheit wurden Schulleitungen als distanzierte Autoritätsfiguren gesehen.

Kathleen: Der erste [Schulleiter] war ein richtiger "Gentleman Head». Er war strikte dagegen, dass Frauen Hosen tragen. Eine Lehrerin hatte eine Hüftoperation und musste um Erlaubnis fragen, Hosen zu tragen, denn das wäre einfacher für sie für die Hüfte (Feldnotizen, Eg, Feb 2000).

Heute wählen Schulleitungen einen eher kollegialen Stil im Umgang mit den Lehrpersonen. Die Sekretärin der kleinen englischen Schule fasst die Veränderung über die Jahre ihrer Tätigkeit so zusammen:

Donna: David ist ein moderner Schulleiter. Den ersten sprach man mit «Sir» an, den zweiten mit "Mister.» Und jetzt mit David verwenden wir Vornamen (Feldnotizen, Ek, Dez. 1999).

Diese Ausschnitte illustrieren die Tradition von machtvollen und häufig autoritären Schulleitungen in England. Die Beschreibung der Lehrpersonen über den früheren Führungsstil wecken Assoziationen von Schulleitern als Monarchen oder Patriarchen.

Die traditionellen hierarchisch strukturierten Machtverhältnisse in englischen Schulen bestehen weiter, wie an späterer Stelle dokumentiert werden wird, geändert haben sich nur die Umgangsformen sowie eine stärkere Betonung des Managements der Schulleitungen, da Schulleitungen mehr Aufgaben und Kompetenzen im Bereich von Finanzen und Administration erhielten. Diese Verschiebung der Rolle der Schulleitung von einer Art «Oberlehrer» zum «Manager» (McHugh \& McMullan, 1995) wird nicht nur positiv wahrgenommen:

David [Schulleiter]: All dieser Bürokram, ich kann es manchmal nicht ausstehen, wenn die Post kommt, mit all diesen Briefen vom Departement und vom Bezirk: Ich fühle mich manchmal hier [im Büro] gefangen [...]. Wir werden immer mehr zu Managern (Feldnotizen, Ek, März 2000).

\section{Veränderung der kollegialen Traditionen in der Schweiz}

In Schweizer Primarschulen wurde die Schulleitung bis vor wenigen Jahren von Klassenlehrpersonen übernommen als zusätzliche administrative Funktion, ohne Einfluss im Gebiet der Personalführung und ohne besondere repräsentative Funktionen gegenüber der Öffentlichkeit oder den Eltern. Schulleitungen wurden häufig auf Grund der Nomination der Lehrpersonen gewählt. Innerhalb der New Public Management-Reformen werden neue Führungsmodelle vorgeschlagen. Oft wird dabei das Prinzip vertreten, dass die Aufgabenbereiche und Kompetenzen der neuen Schulleitungen durch das Team der Lehrpersonen mit Rücksicht auf die lokalen Situationen und Bedürfnisse gestaltet werden sollen und dass die Lehrpersonen als Team Mitsprache bei der Auswahl der Schulleitung haben sollen (Brägger, 1995; Strittmatter, 1996).

Im Kanton Luzern wurde durch die Gesetzgebung von allen Schulen gefordert, dass sie Schulleitungen einsetzen: 
$\$ 44$ Organisation [...] Die Schulleitung ist für die pädagogische und betriebliche Leitung, Führung und Entwicklung der Schule verantwortlich [...]. S 47 Die Schulpflege [...] wählt die Schulleitung» (Luzern, 1999).

Das Team der Lehrpersonen hat nach Gesetz kein Recht auf Mitsprache bei der Auswahl der Schulleitung. Ein Vertreter der Personalverbände kritisiert deshalb: "Schulen mit Profil» brachte im Bereich der Schul-und Schulhausleitungen eine stärkere Hierarchisierung. [...] Der VPOD (Verband des Personals der öffentlichen Dienste, Anm. FV) verlangt mehr demokratische Teilhabe der Lehrpersonen: Das Team schlägt die Schulhausleitung vor, es hat ein Mitspracherecht bei der Wabl der Schulleitung, es wird bei der Erarbeitung des Pflichtenhefts von Schul- bzw. Schulhausleitung einbezogen. Die Amtsdauer der Leitenden soll beschränkt werden (Hug, 2001, S. 30).

Auch in den Schulen der Fallstudien verlangte die Einführung von Schulleitungen Anpassungen für alle Beteiligten. Beide Schulleiter sprachen über die Schwierigkeiten ihrer neuen Rolle. Christoph strebte an, ausschliesslich Schulleiter ohne Verantwortung als Klassenlehrer zu werden.

Christoph: Der Rollenkonflikt, den will ich nicht mehr, den kann ich nicht mehr haben, ich kann nicht Kollege sein und Schulleiter. Ich kann nicht personell beraten, und eventuell sanktionieren, ich kann nicht diese Funktion haben und mit dem gleichen Kollegen den Unterricht vorbereiten. Für mich geht das nicht» (Interview Transkript, $\mathrm{Sg}$ ).

Der Schulleiter der kleinen Schweizer Schule hält es für problematisch, dass er Schulleiter wurde, nachdem er viele Jahre an der Schule unterrichtet hatte.

Andi: Ich merkte schon damals, dass es nicht gut ist. Denn ein guter Schulleiter sollte wirklich von ausserhalb kommen. Man muss die bestehenden Strukturen aufbrechen und das war etwas, das ich eher fürchtete (Interview Transkript, Sk). Beide Schulleiter würden es vorziehen, wenn die Distanz zwischen Schulleitung und Lehrpersonen grösser wäre, indem die Schulleitung nicht als Kollege gesehen würde oder von ausserhalb in die Schule käme. Im Gegensatz dazu sehen die Lehrpersonen die Schulleitung eher als Kollegen.

FV: Empfindest du die Schulleitung als Kollegen und Kolleginnen oder ist da ein gewisser Unterschied? [...] Luzia: Ja, schon, also das ist jetzt für mich nicht irgendwie [...] so der Chef oder so (lacht). [...] Aber ich denke durch das, dass Christoph natürlich vorher auch Schule gegeben hat, ist es einfach ganz anders, ist es einfach, ja wie ein Kollege. FV: Wie wäre das jetzig, wenn, sagen wir, eine Schulleitung von aussen hineinkäme, also wenn das jetzt nicht vorher dein Kollege war, würdest du dich trotzdem immer noch [...]. Luzia: Ich denke das ist sicher schwieriger, wenn du diese Person nicht kennst, und nicht weisst, wie sie ist [...] ich denke, das kann vielleicht schon ein wenig distanzieren voneinander" (Interview Transkript, Sk).

In der Folge von New Public Management-Reformen wurde die Rolle der schweizerischen Schulleitung von einem kollegialen Auftrag zu einer Manage- 
mentfunktion verändert. Schweizer Schulleitungen finden sich im Spannungsfeld zwischen Kollegialität und Managementfunktion; innerhalb der Schulkultur ist ihre Macht erst im Aufbau begriffen. Im Vergleich dazu haben englische Schulleitungen traditionell eine machtvolle Position inne gehabt, die als hierarchische Unterschiede zwischen ihnen und den Lehrpersonen verstanden wurde.

\section{Starke Teams oder starke Schulleitungen?}

Wie wirken sich die oben skizzierten Veränderungen im Machtgefüge der Schulen im Lehrpersonenalltag aus? Welchen Einfluss haben das Team der Lehrpersonen oder die Schulleitungen?

\section{Das Team der Lehrpersonen behält sich demokratische Teilnahme vor}

In der kleinen Schweizer Schule stellte das Lehrpersonenteam und die Schulpflege in der Umsetzung der Reform und der Einführung der Schulleitung ein Organigramm der verschiedenen Funktionen auf. Darin wird detailliert festgehalten, welche Personen (Team der Lehrpersonen, Klassenlehrpersonen, Schulleitung, schulische Heilpädagogin und Schulpflege) informiert werden sollen, welche das Recht zur Mitsprache haben oder welche eine bestimmte Entscheidung fällen können. Die neue Funktion der Schulleitung wurde also bis ins Detail definiert und in ein ausgefeiltes System von Machtüberprüfung eingebettet. Die Teamsitzung der Lehrpersonen wird häufig als souverän in der Entscheidungsfindung der Schule gesehen (Strittmatter, 1996) - nur die Schulpflege und der Gemeinderat werden als hierarchisch übergeordnet wahrgenommen, jedoch nicht die Schulleitung.

In beiden Schweizer Schulen der Fallstudien verlangte das Team, dass sein Einfluss und seine Beziehung zur Schulpflege gestärkt werde. Nach dem Schulleitungsmodell wird die Kommunikation zwischen Schulpflege und Lehrpersonen durch die Schulleitung gewährleistet. Dennoch verlangten die Lehrpersonen der grossen Schweizer Schule ein direktes Treffen zwischen einer Delegation von Lehrpersonen mit einer Delegation der Schulpflege. Während der Teamsitzung informierte eine Lehrerin die anderen über dieses Treffen:

Therese informiert über das Treffen mit der Schulpflege. Eine Mängelliste für die Räume wird durch den Schulleiter der Schulpflege übergeben. Aufteilung der Schulpflegeressorts bekommen wir schriftlich mitgeteilt. Im August, nach Schulpflegetagung orientiert uns die Schulpflege definitiv. Nächstes Treffen mit Schulpflege am 13. September (Anliegen aus dem Team bitte frühzeitig melden) (Felddokument Teamsitzung, Sg, Mai 2000).

Die Analyse der Themen, die zwischen der Delegation von Lehrpersonen und der Schulpflege diskutiert wurden, ohne dass die Schulleitung anwesend war, zeigt, dass diese Aufgaben normalerweise im Kompetenzbereich der Schulleitung 
liegen würden. Aus der Perspektive von englischen Lehrpersonen wäre ein solches Vorgehen sehr ungewöhnlich.

Einer der Schulleiter verwendet einen scheinbar kollegialen, jedoch leicht manipulativen Führungsstil. Das Team ist bei jeder grösseren Entscheidung miteinbezogen. Der Schulleiter führt die Diskussionen jedoch in einer Art und Weise, dass das Ergebnis seinen Zielen entspricht. Mehrere Lehrpersonen kritisieren an der Teamsitzung die fehlende Transparenz:

Nella: Christoph, da dünkt es mich eher das Problem, dass er manchmal nicht wagt. FV: Du möchtest dass er mehr sagt? Nella: Klarer, einfach dass er es sich bewusst ist, will ich das, dass ich das entscheide, oder will er uns die Freiheit geben, dass wir als Team das entscheiden. Und jetzt macht er manchmal so etwas zwischendurch [...] dann sagt er, ja wir tun über das diskutieren, und am Schluss [...] merkst du, eigentlich ist das genau das, was er hätte wollen [...] wir haben ja jetzt heute dieses Gespräch gehabt mit ihm, also wir sind zu dritt, die von der Gruppe her den Auftrag haben, mit ihm über das zu reden (Interview Transkript, $\mathrm{Sg}$ ).

Die Lehrpersonen nehmen die Spannung zwischen hierarchischem Führungsanspruch durch die neue Funktion der Schulleitung und die Tradition der Kollegialität wahr. Das Team behält sich echte Mitsprache vor. Die kollegiale Tradition prägt die Organisationskultur und schränkt den Einfluss der Schulleitung ein, obwohl die neuen Strukturen eine stärkere Führung vorsehen.

\section{Vergleich kritischer Prozesse in der Schulorganisation}

Um die Kräfteverhältnisse zwischen englischen und schweizerischen Schulleitungen und Lehrpersonen zu analysieren, fokussierte ich den Vergleich auf kritische Organisationsprozesse. Ein solcher ist die Stunden- und Klasseneinteilung für die Lehrpersonen. Der Vergleich zwischen den zwei grossen Schulen zeigt starke Unterschiede.

An der grossen englischen Schule wissen auch zwei Wochen vor Schuljahresende mehrere Lehrpersonen noch nicht, welche Klasse sie nach den Sommerferien unterrichten werden.

Beatrice: Falls nicht das Gleiche wieder passiert (die Schulleitung befahl zwei Lehrpersonen zu tauschen gegen deren Wunsch), werde ich in diese Klasse zurückgehen, soviel ich weiss. Falls gewechselt werden muss, ist es die Entscheidung der Schulleitung. Sie kann dich irgendwohin einteilen. Sie hat diese Macht. Letztes Jahr haben einige Lehrpersonen gewechselt. Die Lehrperson der ersten Klasse ging zum Kindergarten und die Kindergartenlehrperson zum ersten und zweiten Jahr und den Entscheid fällte Tina (Schulleiterin). Die Schulleiterin machte diese Entscheidung und ich weiss nicht, was dahinter steckte (Feldnotizen, Eg, Juli 2000).

Die Schulleiterin der grossen englischen Schule setzt ihre Macht und Autorität ein, ohne die betroffenen Lehrpersonen mit einzubeziehen. Die Lehrpersonen sind sich bewusst, dass allein die Schulleitung entscheidet, welche Klasse sie im 
nächsten Jahr unterrichten werden. Die Schulleitung muss die Wünsche der Lehrpersonen nicht berücksichtigen.

An der grossen Schweizer Schule führte die Klasseneinteilung auch zu einem Konflikt. Zwei Teams von Lehrpersonen im Job-Sharing wollten beide die 5 . Klasse unterrichten, es musste jedoch ein Team in die 3. und 4. Klasse wechseln. Christoph (Schulleiter) zeigt mir das Formular vom 2/12, auf dem die Lehrpersonen angeben mussten, ob sie beabsichtigen an der Schule zu bleiben oder in Betracht ziehen zu gehen. Christoph: Es gab grosse Probleme dieses Jahr, weil niemand bereit ist zu wechseln und eine Doppelstelle zu übernehmen. Es kam darauf hinaus, dass beide, Felicitas/Claudia und BettinalJacqueline 5. oder 6. Kl. wollten. Christoph hatte eine Sitzung mit denen [...] angesetzt [...]An der Sitzung gab es keine Lösung [...]. Jedoch hatte Jacqueline signalisiert, dass sie bereit wäre zu wechseln [...]. Bettina war geschockt, sie fühlte, Jacqueline sei ihr in den Rücken gefallen [...]. Es gab keine Einigung und es wurde gesagt, ich solle entscheiden[...]. Die Sitzung endete um 17.10 h und ich sagte, ich würde allen um 18.00 $h$ anrufen und den Entscheid mitteilen. FV: Du wusstest schon, was dein Entscheid ist. Christoph: Ja aber ich musste noch die Begründung formulieren. Die Begründung war, dass Felicitas und Claudia schon länger hier arbeiten [...]. FV: Hat das ungute Gefühle hinterlassen? Christoph: nicht zwischen mir und Bettina aber zwischen den beiden, Jacqueline und Bettina. Es wurde nachher auch angetönt, dass ein umgekehrter Entscheid viele im Team vor den Kopf gestossen hätte und nicht verstanden worden wäre (Feldnotizen, Sg, März 2000).

Dieser Schulleiter bat alle Lehrpersonen, ihre Absichten und Wünsche frühzeitig in einem vertraulichem Formular anzugeben. Mit diesem Vorgehen zeigt der Schulleiter nicht nur, dass jede Lehrperson die gleiche Chance haben sollte, um ihre Wünsche in einem fairen Prozess auszudrücken, sondern auch, dass die Personalführung jetzt in seinen Aufgabenbereich fällt. In der Problemlösung des Konfliktes zwischen Wünschen von verschiedenen Lehrpersonen handelt der Schulleiter zuerst als Mediator und Koordinator und entscheidet nur, nachdem die Lehrpersonen selber realisiert haben, dass sie keinen Konsens erreichen können. In seiner Entscheidung berücksichtigt er die ungeschriebenen Gesetze des Teams wie beispielsweise die Dienstjahre in der Gemeinde.

Im Vergleich dieser zwei Arten von Klasseneinteilung werden die unterschiedlichen Machtverhältnisse in englischen und schweizerischen Schulen sichtbar. Währenddem beide Schulleitungen der grossen Schulen schlussendlich die Entscheidungen in ihrer Funktion alleine trafen, war der Prozess der Entscheidungsfindung jedoch sehr unterschiedlich. Die Schulleiterin der grossen englischen Schule kann sich auf die Macht stützen, Veränderungen zu verordnen, wenn sie möchte, und die resignierte Akzeptanz der Lehrpersonen zeigt, dass diese nicht erwarten, dass sie dabei eine Mitsprache haben. Im Vergleich dazu übergeben die Schweizer Lehrpersonen die Macht der Entscheidung dem Schulleiter erst als letzten Ausweg, in den Fällen, in denen sie selber keinen Konsens finden können. 


\section{Schlussfolgerung: Das politische System und die Organisationskultur prägen die Umsetzung}

Die unterschiedlichen politischen Systeme in England und der Schweiz beeinflussen die Umsetzung der New Public Management-Reformen auf der Ebene des Bildungssystems wie auch in der einzelnen Schule.

Auf der Ebene des Bildungssystems zeigen sich die Unterschiede darin, welche Aspekte des New Public Management betont werden und wie die Reformen eingeführt werden. Um in den Parlamenten und bei einem allfälligen Referendum eine Chance zu haben, müssen die kantonalen Behörden in der Schweiz Vorlagen ausarbeiten, die auf Konsens beruhen. Die nationale Regierung in England hingegen ist unter Druck, in kürzester Zeit Ergebnisse zu zeigen und kann dabei aber auch auf eine grosse Mehrheit im Parlament bauen. In der Folge verwendet die englische nationale Regierung eher Strategien von Befehl und Kontrolle mit nur sehr begrenzter Mitsprache der Lehrpersonen. Die New Public Management-Reform im Kanton Luzern hingegen wurde von der Regierung als Partnerschaft zwischen dem Kanton, den lokalen Schulbehörden und den Berufsverbänden der Lehrpersonen konzipiert. Durch die breitere Mitsprache gab es wichtige Kompromisse auf allen Seiten. Die Unterschiede wirkten sich direkt darauf aus, in welchem Tempo und Umfang die New Publik Management-Reform eingeführt wurden: In der Schweiz haben die Reformen langsam und gemässigt eingesetzt, während das englische System viel umfassender und in kürzerer Zeit verändert wurde.

Die Unterschiede im politischen System wirken sich auch auf der lokalen Ebene aus und prägen die Organisationskultur in den Schulen, wie am Beispiel des Machtverhältnisses zwischen dem Team der Lehrpersonen und den Schulleitungen gezeigt wurde. Die Reformen des New Public Management stärken in beiden Ländern die Funktion der Schulleitungen. Obwohl die Schulleitungen im Kanton Luzern auf Grund der neuen Gesetzgebung eine stärkere, machtvollere Position einnehmen könnten, erlaubt es ihnen die Organisationskultur nicht, diese auch auszuüben. Die Teams beider Fallstudienschulen sorgen dafür, dass die starke kollegiale Tradition weiterbesteht. Die Lehrpersonen tendieren dazu, ihr Team als souveränes oder übergeordnetes Gremium für die Entscheide der Schule zu sehen, welches nur durch die lokalen Schulbehörden und den Gemeinderat der politischen Gemeinde überstimmt werden kann, jedoch nicht durch die Schulleitung. Lehrpersonen zögern nicht, lokale politische Strukturen zu nutzen, um Einfluss nehmen zu können. Hier hat die Kultur, jedenfalls zu diesem Zeitpunkt der Reform, den Einfluss der Schulreform und der Gesetzesänderungen gehemmt. Das Team der Lehrpersonen ist in keiner Weise in einem legalen Sinne souverän, die neuen Schulleitungen haben Machtbefugnisse im Management der Schule. Trotzdem wäre es für die Schulleitungen schwer möglich, ihren Willen gegen die Mehrheit der Lehrpersonen durchzusetzen. Im Gegensatz dazu können die englischen Schulleitungen auf eine lange Tradition 
starker Schulleitungen mit hierarchischen Schulkulturen bauen, ohne auf die Wünsche der Lehrpersonen eingehen zu müssen. Die New Public ManagementReformen konnten auf den existierenden Machtverhältnissen aufbauen und haben die Differenzierung zwischen den Rollen der Schulleitungen als Managers und den Lehrpersonen als deren Mitarbeitende verstärkt.

Die Fallstudien weisen darauf hin, dass politische Systeme die Umsetzung der Reformen auch auf der Ebene der lokalen Schule beeinflussen. Die Fallstudien lassen sich jedoch nicht empirisch generalisieren. Darum ist weitere Forschung nötig, um die Interaktion zwischen dem politischen System eines Landes, der Organisationskultur in den Schulen und der Umsetzung der Reformen auf der lokalen Ebene breiter zu untersuchen. Vergleichenden Analysen zwischen verschiedenen Ländern, die auch den Einfluss des Systems der politischen Entscheidungsfindung miteinbeziehen, können einen Beitrag leisten, um die Forschung zu Organisationskultur und Schulreform weiter zu führen.

\section{Anmerkung:}

Mein Dank geht an die Lehrpersonen aller vier Primarschulen, die an diesem Forschungsprojekt teilgenommen haben und an den Schweizerischen Nationalfonds für die finanzielle Unterstützung.

\section{Literaturverzeichnis}

Alford, J. (1993). Towards a New Public Management Model: Beyond «Managerialism» and its Critics. Australian Journal of Public Administration, 52, (2), 135-148.

Alvesson, M. \& Deetz, S. (1999). Critical Theory and Postmodernism: Approaches to Organizational Studies. In S.R. Clegg \& C. Hardy (Hrsg.), Studying Organization. Theory and Method (S. 185-211). London: Sage.

Ball, S.J. (1998). Big Policies/Small World: an Introduction to International Perspectives in Education Policy. Comparative Education, 34, (2), 119-130.

Bottery, M. (2000). Education, Policy and Ethics. London: Continuum.

Clarke, J. \& Newman, J. (1993). The Right to Manage: a Second Managerial Revolution? Cultural Studies, 7, (3), 427-441.

Clarke, J. \& Newman, J. (1997). The Managerial State: Power, Politics and Ideology in the Remaking of Social Welfare. London: Sage.

Deem, R. (1998). «New Managerialism» and Higher Education: the Management of Performances and Cultures in Universities in the United Kingdom. International Studies in Sociology of Education, 8, (1), 47-70.

Deem, R., Brehony, K.J. \& Heath, S. (1995). Active Citizenship and the Governing of Schools. Buckingham: Open University Press.

Exworthy, M. \& Halford, S. (1999). Professionals and Managers in a Changing Public Sector: Conflict, Compromise and Collaboration? In M. Exworthy \& S. Halford (Hrsg.), Professionals and the New Managerialism in the Public Sector (S. 1-17). Buckingham: Open University Press.

Farnham, D. \& Horton, S. (1993). The New Public Service Managerialism: An Assessment. In D. Farnham \& S. Horton (Hrsg.), Managing the New Public Services (S. 237-254). Basingstoke: Macmillan. 
Ferlie, E., Ashburner, L., Fitzgerald, L. \& Pettigrew, A. (1996). The New Public Management in Action. Oxford: Oxford University Press.

Flynn, R. (1999). Managerialism, Professionalism and Quasi-Markets. In M. Exworthy \& S. Halford (Hrsg.), Professionals and the New Managerialism in the Public Sector (S. 18-36). Buckingham: Open University Press.

Gomm, R., Hammersley, M. \& Foster, P. (2000). Case Study and Generalization. In R. Gomm, M. Hammersley \& P. Foster (Hrsg.), Case Study Method. Key Issues, Key Texts (S. 98-115). London: Sage.

Hug, H.-P. (2001). «Schulen mit Profil» ( Zwischenbilanz des VPOD). VPOD Magazin, (123), 30-31.

Kogan, M. (1986). Education Accountability. An Analytic Overview. London: Hutchinson.

Lowe, G. (1998). Inspection and Change in the Classroom: Rhetoric and Reality? In P. Earley (Hrsg.), School Improvement after Inspection? School and LEA Responses (S. 97-109). London: Paul Chapman.

Luzern. (1999). Gesetz über die Volksschulbildung (No. Nr 400a). Luzern: Bildungsdepartement des Kantons Luzern.

McHugh, M. \& McMullan, L. (1995). Headteacher or Manager? Implications for Training and Development. School Organisation, 15, (1), 23-34.

Mill, J.C.H., \& Mills, A.J. (2000). Rules, Sensemaking, Formative Contexts, and Discourse in the Gendering of Organizational Culture. In N.M. Ashkanasy, C.P.M. Wilderom \& M.F. Peterson (Hrsg.), Handbook of Organizational Culture and Climate (S. 55-70). Thousand Oaks: Sage.

Pollitt, C. \& Bouckaert, G. (2000). Public Management Reform. A Comparative Analysis. Oxford: Oxford University Press.

Schein, E.H. (1991). What is Culture? In P.J. Frost, L.F. Moore, M.R. Louis, C.C. Lundberg \& J. Martin (Hrsg.), Reframing Organizational Culture (S. 243-253). Newbury Park: Sage.

Schofield, J.W. (2000). Increasing the Generalizability of Qualitative Research. In R. Gomm, M. Hammersley \& P. Foster (Hrsg.), Case Study Method. Key Issues, Key Texts (S. 69-97). London: Sage.

Skeggs, B. (1994). Situating the Production of Feminist Ethnography. In M. Maynard \& J. Purvis (Hrsg.), Researching Women's Lives from a Feminist Perspective (S. 72-92). London: Taylor \& Francis.

Trowler, P. (1998). What Managerialists Forget: Higher Education Credit Frameworks and Managerialist Ideology. International Studies in Sociology of Education, 8, (1), 91-109.

Vogt, F. (2001). Researching the Impact of New Public Management Approaches to Policy on Primary School Teachers» Work. In G. Walford (Hrsg.), Ethnography and Education Policy (S. 137-156). Oxford: Elsevier Science.

Vogt, F. (2002). Contextualising New Managerial Policy Enactment in Primary Schools in England and Switzerland: Teacher's Perceptions and Experiences of Policy Change, Organisational Culture and Teamwork. Unpublished PhD, Lancaster University, Lancaster.

Vogt, F. (2002). No Ethnography without Comparison: The Methodological Significance of Comparison in Ethnographic Research. In G. Walford (Hrsg.), Debates and Developments in Ethnographic Methodology (S. 23-42). Oxford: Elsevier Science.

Schlagworte: Bildungspolitik, Schulautonomie, Schulleitung, Schulaufsicht, Ländervergleich, New Public Management 


\section{Système politique et New Public Managment dans les écoles primaires en comparaison internationale.}

\section{Résumé}

Le but de cette enquête sur la réforme scolaire et sa mise en œuvre est l'exploration du jeu qui se met en place entre l'idéologie internationale du New Public Management et les systèmes politiques locaux et les cultures professionnelles. L'Angleterre et la Suisse permettent une comparaison particulièrement intéressante, car leurs systèmes politiques se différencient fortement - centralisé et en Majorsystem en Angleterre, décentralisé et construit sur un consensus recoupant différents partis en Suisse (Politt \& Bouckaert, 2000). Cet article pose la question de savoir comment le travail commun parmi les professeurs et le rôle de la direction de l'école sont transformés. Les résultats de quatre études de cas indiquent que le système politique et la modalité de la réforme scolaire influent non seulement sur le plan du système de formation, mais aussi sur le plan spécifique à chaque école, tout particulièrement dans les processus de décisions scolaires et dans les structures de pouvoir entre direction des écoles, autorités scolaires locales et professorat.

Mots clefs: Politique de la formation, autonomie de l'établissement scolaire, direction d'école,surveillance de l'établissement scolaire, comparaison entre pays, New Public Management

\section{Sistema politico e New Public Management nelle scuole elementari - un confronto internazionale}

\section{Riassunto}

L'obiettivo di questa analisi della riforma scolastica è l'esplorazione del rapporto tra l'ideologia transnazionale del New Public Management e i sistemi politici locali con le loro culture professionali. L'Inghilterra e la Svizzera permettono un confronto particolarmente interessante perché dispongono di sistemi politici molto diversi - centralizzato e basato sul maggioritario quello inglese, decentralizzato e basato su un ampio consenso dei partiti politici quello svizzero (Pollitt $\&$ Bouckeart, 2000). L'articolo affronta la questione del rapporto tra gli insegnanti e il ruolo delle direzioni scolastiche. I risultati emersi dall'analisi di quattro casi mostrano come il sistema politico caratterizzi la riforma scolastica non solo a livello di sistema scolastico, ma anche a livello delle singole scuole, in particolare per quanto attiene ai processi decisionali e ai rapporti di potere tra direzione scolastica, amministrazione locale della scuola e insegnanti.

Parole chiave: Politica formativa, amministrazione scolastica, autonomia scolastica, direzione delle scuole, sorveglianza scolastica, confronto tra stati, New Public Management 


\title{
Political system and New Managerialism in primary schools - an international comparison
}

\begin{abstract}
The analysis of new managerial policy change in two different countries enables the exploration of the interplay between an ideology dominant across many countries and the local political system and professional culture. England and Switzerland provide an interesting comparison because of the differences of their political system - majoritarian and centralised in England, consensual and decentralised in Switzerland (Pollitt \& Bouckaert, 2000). In this article changes in teachers' teamwork and in the headteacher's role are examined. Based on a research project involving four case studies, it is argued that the political system influences the enactment of new managerial reform not only at the level of the education system but also at the level of the local school, in particular in relation to the decision making processes and the power relations between management, school governing body and teachers.
\end{abstract}

Key words: educational policy, school atonomy, school director, school board, comparison of countries, New Public Management 
T h e m a 